American Journal of Infectious Diseases 5 (1): 7-10, 2009

ISSN 1553-6203

(C) 2009 Science Publications

\title{
Detection of Mycoplasma genitalium and Trichomonas vaginalis Infections in General Jordanian Patients
}

\author{
${ }^{1}$ A.A. Shehabi, ${ }^{2}$ Z.M. Awwad, ${ }^{3}$ M. Al-Ramahi, ${ }^{4}$ E. Charvalos and ${ }^{1}$ L.F. Abu-Qatouseh \\ ${ }^{1}$ Departments of Pathology and Microbiology \\ ${ }^{2}$ Special Surgery-Urology \\ ${ }^{3}$ Obstetric and Gynecology \\ Jordan University Hospital, Amman, Jordan \\ ${ }^{4}$ Institute of Microbiology, University of Greece, Athena, Greece
}

\begin{abstract}
Problem statement: Both M. genitalium and T. vaginalis were recognized as important cause of sexually transmitted infections in developed countries. This study investigated the prevalence of M. genitalium and T. vaginalis in general Jordanian patients and their role of causing genitourinary tract diseases. Approach: A cross sectional study of 383 Jordanian adult patients aged between 19-78 years were investigated for presence of M. genitalium and T. vaginalis at the urology and obstetric-gynecology clinics at the Jordan University Hospital in Amman. First voided urine specimens were tested using urine microscopy, PCR for M. genitalium and T. vaginalis as well as culture for $T$. vaginalis. Results: The incidence of $M$. genitalium was higher and statistically significant $(17 / 188,9 \%, \mathrm{p}=0.022)$ than $T$. vaginalis $(3 / 188 ; 1.6 \%)$ among patients diagnosed with specific urinary symptoms and signs, while this incidence was less but also significant in asymptomatic patients $(7 / 195,3.6 \%$ versus $1 / 195,0.5 \%, \mathrm{p}=0.031)$, respectively. M. genitalium infection was frequently observed with urinary frequency $(76 \%)$ and dysuria (59\%) among symptomatic patients and more common in men than women $(65 \%$ versus $35 \%$, $\mathrm{p}=0.51)$ and in married than singles $(76 \%$ versus $24 \%, \mathrm{p}=0.59)$. Dual infection with both organisms was not recognized. Conclusion: Infection caused by $M$. genitalium and $T$. vaginalis was associated with higher incidence rate in patients with symptomatic genitourinary disease. Therefore, screening for their occurrence in such patients is important.
\end{abstract}

Key words: $M$. genitalium, $T$. vaginalis, urine PCR, Jordan

\section{INTRODUCTION}

Nongonococcal Urethritis (NGU) caused by Chlamydia trachomatis Mycoplasma genitalium and Trichomonas vaginalis are common cause of symptomatic and asymptomatic infections in both men and women in developed countries ${ }^{[1,4]}$ but their prevalence and pathogenesis in most developing countries including Jordan are still limited reported ${ }^{[5,6] .}$

Recently, M. genitalium has been recognized as a common infection associated with symptomatic urethritis and with a high prevalence of infected sexual partners supporting its role as a sexually transmitted infection $^{[1,4,7,8]}$. First voided urine appeared to be a better diagnostic specimen than the urethral swab for detection M. genitalium in men using $\mathrm{PCR}^{[8-10]}$. In women, M. genitalium cause cervisitis, urethritis, pelvic disease and recently found more commonly in cervical canal of infertile women and its infection can be also detected with high sensitivity by using urine specimen and $\mathrm{PCR}^{[7,9-11]}$.
Epidemiologically, $T$. vaginalis infection is often associated with vaginosis and commonly transmitted with other STDs, whereas its prevalence and spectrum of disease in men are less characterized ${ }^{[12,13]}$. Diagnosis of $T$. vaginalis is usually made using wet mount of vaginal swabs and direct microscopy, which are not highly sensitive, while culture method gives results that are more positive but it is less performed. Recent studies showed that detection of $T$. vaginalis using urine or urethral swab specimens and PCR showed high sensitivity and positive results ${ }^{[13-15]}$.

The purpose of this study was to determine the rate of infection with $M$. genitalium and $T$. vaginalis and their association with common specific genitourinary features in general Jordanian patients.

\section{MATERIALS AND METHODS}

Patients: A total of 383 Jordanian patients aged between 19-78 years, including 201 (52\%) men with

Corresponding Author: Asem A. Shehabi, Departments of Pathology-Microbiology, Jordan University Hospital, Amman, Jordan 
Am. J. Infect. Dis., 5 (1): 7-10, 2009

Table 1: Distribution of patients and controls with and without specific genitourinary symptoms and positive T. vaginalis and M. genitalium

\begin{tabular}{|c|c|c|c|c|c|}
\hline \multirow{2}{*}{ 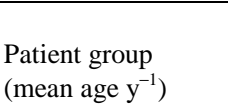 } & \multicolumn{2}{|c|}{ Sex } & \multirow[b]{2}{*}{ Positive $T$. vaginalis $\mathrm{N}(\%)$} & \multirow[b]{2}{*}{ Positive M. genitalium N (\%) } & \multirow[b]{2}{*}{ Total } \\
\hline & Males N (\%) & Females & & & \\
\hline Symptomatic $^{\mathrm{a}}$ (42) & $99(53)$ & $89(47)$ & $3(1.6)$ & $17(9.0)$ & 188 \\
\hline Asymptomatic (40) & $102(52)$ & $93(48)$ & $1(0.5)$ & $7(3.6)$ & 195 \\
\hline Total & $201(52)$ & $182(48)$ & $4(1.0)$ & $24(6.2)$ & 383 \\
\hline
\end{tabular}

${ }^{a}$ : Each patient complained of one or more specific genitourinary symptoms (urinary frequency, dysuria, suprapubic/pelvic pain, presence of vaginal/urethral discharge )

mean age of 42-year and $182(48 \%)$ women with mean age of 40-year were admitted at the urology and obstetric-gynecology clinics at the Jordan University Hospital (JUH) in Amman, over the period MayOctober 2006. All patients were examined for the presence of any of the following specific urinary symptoms: urinary frequency, dysuria, suprapubic/pelvic pain and presence of vaginal/urethral discharge. Of these, 188 were characterized as symptomatic patients with one or more specific genitourinary symptoms and signs and the rest 195 subjects were free of any specific urinary symptoms and have been included as controls (Table 1). All Patients gave their written consent to be included in this study and the clinical data of each enrolled patient were reported in a special designed form for the study.

Urine specimen and culture of $\boldsymbol{T}$. vaginalis: First void urine specimens were collected in sterile leak proof containers from all patients included in the study. Urine specimens were transported to the microbiology research laboratory for investigation with $2 \mathrm{~h}$, at the department of pathology and microbiology, Faculty of Medicine, University of Jordan. Ten $\mathrm{mL}$ of urine specimens were centrifuged at $2000 \mathrm{~g}$ for $10 \mathrm{~min}$ and the pellet was examined microscopically for the presence of the motile trophozoit of $T$. vaginalis and to count White Blood Cells (WBCs) per High Power Field (HPF). The pellet was then resuspended in $1 \mathrm{~mL}$ of Phosphate Buffer Saline (PBS) and $0.5 \mathrm{~mL}$ of the sample kept at $-70^{\circ} \mathrm{C}$ for later $\mathrm{PCR}$ investigation, whereas the second $0.5 \mathrm{~mL}$ was inoculated in a vial containing $5 \mathrm{~mL}$ of Trichomonas medium No.2 (Oxoid, UK). The culture media were incubated at $37^{\circ} \mathrm{C}$ up to 7 days and the media was checked daily by microscopic examination for the presence of lived, motile Trophozoits of $T$. vaginalis $^{[13]}$.

DNA extraction and PCR: DNA extraction was performed according to the instructions provided in the Genomic Wizard DNA extraction Kit (Promega, USA). $T$ vaginalis specific primers TVK3 (5`ATTGTCGAACATTGGTCTCCTC 3`) and TVK7 (5 TCTGTCCCGTCT TCAAGTATGC $\left.3^{\prime}\right)^{[13]}$. For $M$. genitalium, $\quad \mathrm{MgPa} \quad 1$ AGTTGATGAAACCTTAACCCCTTGG-3') and
MgPa3 (5'-CCGTTGAGGGGTTTTCCATTTTTGC-3') primers were used for PCR amplification ${ }^{[16]}$. PCR reaction mixture of a total volume of $25 \mathrm{uL}$ was composed of $15 \mathrm{uL}$ of PCR Master Mix (Promega, USA) $2.5 \mathrm{uL}$ of $10 \mathrm{uM}$ each primer and $5 \mathrm{uL}$ of extracted DNA of urine specimens of each patient. The master mix was composed of $3 \mathrm{uM} \mathrm{MgCl}_{2}, 500 \mathrm{uM}$ dNTPs each, 1U Taq DNA polymerase and Taq buffer. Positive and negative controls were included in all PCR runs. The positive control of $T$. vaginalis was composed of DNA extracted from our clinical isolate of $T$. vaginalis which was grown in Trichomonas medium No.2 (Oxoid. UK), where the positive control of $M$. genitalium consisted of DNA provided as lyophilized compound supplied from Institute of Microbiology, University of Greece in Athena (E. Charvalos). Negative control was made of distilled water. PCR amplification was performed in two separate tubes for both organisms as follows: 30 cycles of $1 \mathrm{~min}$ at $90^{\circ} \mathrm{C}, 30 \mathrm{~s}$ at $60^{\circ} \mathrm{C}$ and $2 \mathrm{~min}$ at $72^{\circ} \mathrm{C}$. After amplification, there was additional extension step at $72^{\circ} \mathrm{C}$ for $7 \mathrm{~min}$ and then samples were cooled to $4^{\circ} \mathrm{C}$.

Gel electrophoresis: $15 \mathrm{uL}$ of amplified product was electrophoresed on a $2 \%$ agarose gel containing $0.5 \mathrm{ug}$ $\mathrm{mL}^{-1}$ ethidium bromide and viewed on a gel documentation system (UVP, USA). Samples containing a 300 and 290bp fragments were considered positive for $T$. vaginalis and $M$. genitalium, respectively ${ }^{[14,17]}$.

Statistical analysis: Statistical significance was determined using $\chi^{2}$ and Fisher's exact tests. Results were considered statistically significant if the $\mathrm{p}$ value was $<0.05$.

\section{RESULTS}

The incidence of $M$. genitalium was higher and statistically significant $(17 / 188,9 \%, \mathrm{p}=0.022)$ than $T$. vaginalis $(3 / 188,1.6 \%)$ among symptomatic patients with specific urinary symptoms and signs, whereas this incidence was less but also statistically significant in asymptomatic patients $(7 / 195,3.6 \%$ versus $1 / 195$, 
Am. J. Infect. Dis., 5 (1): 7-10, 2009

Table 2: Clinical and epidemiological features of 3 symptomatic patients and one asymptomatic control person with positive with T. vaginalis Patients

\begin{tabular}{llllcl} 
Sex & Age & Marital status & Specific symptoms & WBC count in urine/ HPF & Laboratory results \\
\hline M & 55 & Married & Dysuria, pelvic pain, orchitis & $15-20$ & +ve PCR -ve Microscopy -ve Culture \\
M & 60 & Married & Dysuria Recurrent urination, & $0-2$ & +ve PCR -ve Microscopy -ve culture \\
M & 55 & Married & Not present & $0-2$ & +ve PCR -ve Microscopy -ve culture \\
F & 36 & Married & Dysuria, pelvic pain & $6-8$ & +ve PCR +ve Microscopy +ve culture \\
\hline
\end{tabular}

Table 3: Clinical and epidemiological features of 17 patients with positive M. genitalium

\begin{tabular}{ll}
\hline Patients & No. $(\%)$ \\
\hline Total No. & $17(100)$ \\
Mean Age & 39.9 years \\
Sex & \\
Males & $11(65)$ \\
Females & $6(35)$ \\
Marital status & \\
Married & $13(76)$ \\
Single & $4(24)$ \\
Urine microscopy & $7(41)$ \\
Numerous WBCs (> 5/ HPF) & $10(59)$ \\
Few WBCs (1-4/ HPF) & \\
Specific symptoms & \\
Urinary frequency & $13(76)$ \\
Dysuria & $10(59))$ \\
Suprapubic/pelvic pain & $6(35)$ \\
Urethral discharge & $1(6)$ \\
\hline${ }^{a}:$ Most patients (13/17, 76\%) have at least two specific genitourinary \\
symptoms
\end{tabular}

$0.5 \%, \mathrm{p}=0.031$ ), respectively (Table 1$)$. Dual infection was not recognized and men to female infection ratio was approximately 2:1 (65\% versus $35 \% \mathrm{p}=0.51)$ for both organisms and was more common in married patients $(76 \%$ versus $24 \%, \mathrm{p}=0.59)$ (Table 2 and 3 ).

Table 2 shows characteristics of $T$. vaginalis infection and detection methods in 3 symptomatic patients and one asymptomatic. Table 3 shows the incidence and characteristics of 17 symptomatic patients associated with positive $M$. genitalium infections. Urinary frequency $13 / 17(76 \%)$ and dysuria $10 / 17(59 \%)$ were frequently recognized as common symptoms among these patients.

\section{DISCUSSION}

Information on the incidence and spectrum of clinical features associated with fastidious organisms like C. trachomatis, M. genitalium and T. vaginalis, is still rarely reported in Jordan and in most Middel East countries. One study reported that $T$. vaginalis has been detected in $0.9 \%$ of women using cervical stained smears over a period of 3.5 years ${ }^{[6]}$, while a study from Egypt showed that trichomoniasis symptomatic cases were detected more by PCR $(91.3 \%)$ than by culture $(72.9 \%)$ or other routine methods ${ }^{[17]}$.

This study indicated that $M$. genitalium is more prevalent and significant than $T$. vaginalis (9\% versus
$1.6 \%, \mathrm{p}=0.022$ ) among Jordanian patients with symptomatic genitourinary infections, respectively, while the incidence of both organisms in a symptomatic control group was less but also significant (3.6 and $0.5 \%, \mathrm{p}=0.031)$, respectively. Dual infection has been not diagnosed and males to female infection ratio was approximately $2: 1$ for both organisms. These results indicate that the incidence of $M$. genitalium in our male and female patients is similar to some extent to recent studies from northern European countries which have reported a range of 6-12\% among their population $^{[7,8,18,19]}$. The overall incidence of $T$. vaginalis infection in Jordanian population either symptomatic $(1.6 \%)$ or a symptomatic $(0.5 \%)$ is much less than that reported from most developed countries using similar clinical specimens and PCR techniques ${ }^{[12-}$ 15].

Most symptomatic patients infected with M. genitalium (76\%) have at least two specific symptoms; urinary frequency and dysuria, whereas about one third of the patients (36\%) suffered from pelvic or suprapubic pain and only one patient has urethral discharge (Table 3). In addition, presence of few pus cells $(59 \%, \mathrm{p}>0.05)$ has be been detected more often than numerous pus cells $(41 \%)$ in urine of infected symptomatic patients (Table 2 and 3). The spectrum of genitourinary symptoms and signs among our patients shows that clinical diagnosis and confirmation of both infections of $M$. genitalium and $T$. vaginalis require full clinical and laboratory investigations. A recent study performed in England, has shown that both urethritis and the presence of a urethral discharge and/or dysuria are significantly associated with the detection of $M$. genitalium $^{[19]}$. This study also found like other studies that detection of $T$. vaginalis using first void urine specimens and PCR performed better than wet mount microscopy and culture, particularly in men with $\mathrm{NGU}^{[1,13,14,20]}$.

\section{CONCLUSION}

Infection with $M$. genitalium and $T$. vaginalis is associated with higher incidence rate in patients with symptomatic genitourinary disease than in asymptomatic. Therefore, screening for their presence in symptomatic patients is important. 
Am. J. Infect. Dis., 5 (1): 7-10, 2009

\section{ACKNOWLEDGMENT}

This study was supported with a grant (No. 5/3/1/1927) from the deanship of academic research, University of Jordan, Amman, Jordan

\section{REFERENCES}

1. Martin, D.H., 2008. Nongonococcal urethritis: New views through the prism of modern molecular microbiology. Curr. Infect. Dis. Rep., 10: 128-132. PMID: 18462587.

2. Massari, V., Y. Dorleans and A. Flahault, 2006. Persistent increase in the incidence of acute male urethritis diagnosed in general practices in France. Br. J. Gen. Pract., 56: 110-114. PMID: 16464324.

3. Miller, W.C., H. Swygard and M.M. Hobbs et al., 2005. The prevalence of trichomoniasis in young adults in the United States. Sex. Trans. Dis., 32: 593-598. PMID: 16205299.

4. Falk, L., H. Fredlund and J.S. Jensen, 2004. Symptomatic urethritis is more prevalent in men infected with Mycoplasma genitalium than with Chlamydia trachomatis. Sex. Trans. Infect., 80: 289-293. PMID: 15295128.

5. Awwad, Z.M., A.A. AL-Amaratand and A.A., Shehabi, 2003. Genital chlamydial infection in symptomatic and asymptomatic Jordanian patients. Inter. J. Infect. Dis., 7: 206-209. DOI: 10.1016/S12019712(03)90053-1.

6. Malkaw, S.R., R.M. Abu Hazeem, B.M. Hajjat and F.K. Hajjiri, 2004. Evaluation of cervical smears at King Hussein medical centre, Jordan, over three and a half years. East Mediterr Health J., 10: 676-679. PMID: 16335662.

7. Falk, L., H. Fredlund and J.S. Jensen, 2005. Signs and symptoms of urethritis and cervicitis among women with or without Mycoplasma genitalium or Chlamydia trachomatis infection. Sex. Trans. Infect., 81:73-78. PMID: 15681728.

8. Ross, J.D. and J.S. Jensen, 2006. Mycoplasma as a sexually transmitted infection: Implication for screening and treatment. Sex. Trans. Infect., 82: 269-271. PMID: 16877571.

9. Jensen, J.S., E. Bjornelius, B. Dohn and P. Lidbrink, 2004. Comparison of first void urine and urogenital swab specimens for detection of Mycoplasma genitalium and Chlamydia trachomatis by polymerase chain reaction in patients attending a sexually transmitted disease clinic. Sex Trans. Dis., 31: 499-507. PMID: 15273584.
10. Jurstrand, M., J.S. Jensen and H. Fredlund et al., 2005. Detection of Mycoplasma genitalium in urogenital specimens by real-time PCR and by conventional PCR assay. J. Med. Microbiol., 54: 23-29. PMID: 15591251.

11. Grześko, J., M. Elias, B. Mączyńska, U. Kasprzykowska, M. Tłaczała, M. Goluda, 2008. Occurrence of Mycoplasma genitalium in fertile and infertile women. Fertil Steril. PMID: 18571169.

12. Schwebke, J.R. and D. Burgess, 2004. Trichomoiasis. Clin. Microbiol. Rev., 17: 794-803. PMID: 15489349.

13. Lawing, L.F., S.R. Hedges and J.R. Schwebke, 2000. Detection of trichomonosis in vaginal and urine specimens from women by culture and PCR. J. Clin. Microbiol., 38: 3585-3588. http://jcm.asm.org/cgi/content/abstract/38/10/3585.

14. Schwebke, J.R. and L.F. Lawing, 2002. Improved detection by DNA amplification of Trichomonas vaginalis in Males. J. Clin. Microbiol., 40: 3681-3683. PMID: 12354865.

15. Wendel, K.A., E.J. Erbelding, L.A. Gaydos and A.M. Rompalo, 2003. Use of urine polymerase chain reaction to define the prevalence and clinical presentation of Trichomonas vaginalis in men attending an STD Clinic. Sex. Trans. Infect., 79: 151-153. PMID: 12690140.

16. Jensen, J.S., S.A. Uldum, J. Sonderfard-Anderson, J. Vuust and L. Klaus, 1991. Polymerase chain reaction for detection of $M$. genitalium in clinical samples. J. Clin. Microbiol., 29: 46-50. PMID: 1993766.

17. Negm, A.Y. and D.A. El-Haleem, 2004. Detection of trichomoniasis in vaginal specimens by both conventional and modern molecular tools. J. Egypt. Soc. Parasitol., 34: 589-600. PMID: 15287181.

18. Anagrius, C., B. Lre and J.S. Jensen, 2005. Mycoplasma genitalium: prevalence, clinical significance and transmission. Sex. Trans. Infect., 81: 458-462. PMID: 16326846.

19. Leung, A., K. Eastick, L.E. Haddon, C.K. Horn, D. Ahuja, P.J. Horner, 2006. Mycoplasma genitalium is associated with symptomatic urethritis. Int. J. Std. AIDS, 17: 285-288. PMID: 16643675.

20. Crucitti, T., E. Van Dyck, A. Tehe, S. Abdellati, B. Vuylsteke, A. Buve and M. Lega, 2003. Comparison of culture and different PCR assays for detection of Trichmonas vaginalis in self collected vaginal swab specimens. Sex. Trans. Infect., 79: 393-398. PMID: 14573835. 